\title{
Desempenho de Estimadores de Volatilidade na Bolsa de Valores de São Paulo*
}

\author{
Bernardo de Sá Mota** \\ Marcelo Fernandes ${ }^{* * *}$
}

Sumário: 1. Introdução; 2. Descrição dos dados; 3. Volatilidade realizada; 4. Modelos da família GARCH; 5. Medidas alternativas de volatilidade; 6 . Avaliação dos estimadores de volatilidade; 7 . Conclusão.

Palavras-chave: Martingal local; valor em risco; variação quadrática; variância realizada.

Códigos JEL: C22; G12.

O objetivo deste artigo é avaliar o desempenho de diferentes métodos de extração da volatilidade do Índice da Bolsa de Valores de São Paulo (IBOVESPA) tendo como referência a volatilidade realizada. Comparamos modelos da família GARCH com estimadores alternativos baseados em cotações de abertura, fechamento, máximo e mínimo. Os resultados indicam que os estimadores alternativos são tão precisos quanto os modelos do tipo GARCH, apesar de serem muito mais econômicos em termos computacionais.

\section{Introdução}

O estudo da volatilidade tem grande importância na área de finanças, em especial no apreçamento de derivativos e no gerenciamento de risco. A literatura de extração de volatilidade inclui métodos com diversos graus de dificuldade de implementação. O estimador mais simples é o desvio padrão histórico, que atribui peso uniforme a todas as observações. Em contraste, o alisamento exponencial (EWMA) aloca peso maior para as observações mais recentes, mas apresenta o inconveniente da escolha arbitrária do grau de suavização. Os modelos da família GARCH e de volatilidade estocástica, por não sofrerem destes problemas, são bastante populares.

${ }^{*}$ Artigo recebido em out. 2002 e aprovado em jul. 2003. Os autores são gratos a Osmani Guillén, João Victor Issler, Hedibert Lopes, Guilherme Rocha e Pedro Saffi pelos comentários e ao apoio financeiro prestado por CAPES, CNPq, FAPERJ, e PRONEX. Qualquer erro é de exclusiva responsabilidade dos autores.

${ }^{* *}$ Máxima Asset Management. E-mail: bernardo_mota@maximaasset.com.br

*** Escola de Pós-Graduação em Economia, Fundação Getulio Vargas. E-mail: mfernand@fgv.br 
Apesar da maioria dos estudos utilizando modelos do tipo GARCH e de volatilidade estocástica encontrarem coeficientes altamente significativos, estes modelos exibem um fraco desempenho em termos de previsão do retorno futuro ao quadrado. Andersen e Bollerslev (1998) demonstram que o problema não reside no poder de previsão dos modelos, mas sim na base de comparação. Apesar de ser um estimador não viesado da variância, o retorno ao quadrado é não apenas altamente volátil, mas também inconsistente. Andersen e Bollerslev sugerem, então, uma base de comparação alternativa denominada variância realizada, que é um estimador assintoticamente livre de erro. Andersen e Bollerslev mostram ainda que os modelos da família GARCH apresentam boas previsões da variância realizada.

Neste artigo, investigamos o desempenho dos estimadores alternativos de Garman e Klass (1980) - doravante estimadores GK - na extração da volatilidade do IBOVESPA através da metodologia desenvolvida por Andersen e Bollerslev (1998). Mais especificamente, mensuramos a precisão das estimativas e previsões de cada estimador GK com base na variância realizada do IBOVESPA. Os estimadores GK têm a facilidade de implementação como principal característica, pois dependem apenas de dados publicados diariamente nas seções de finanças de qualquer jornal especializado: cotações de abertura, fechamento, máximo e mínimo. Para efeito de comparação, examinamos também a acurácia dos modelos do tipo GARCH, usualmente adotados pela literatura. Os resultados são de certa forma surpreendentes, pois indicam que alguns estimadores GK têm desempenho comparável aos dos modelos GARCH.

O restante do artigo está estruturado da seguinte forma. A seção 2 descreve estatisticamente os dados. A seção 3 apresenta a metodologia da volatilidade realizada e a estimativa da volatilidade do IBOVESPA. As seções 4 e 5 apresentam as estimativas dos modelos da família GARCH e as medidas alternativas de volatilidade de Garman e Klass (1980), respectivamente. A seção 6 avalia o desempenho dos diversos estimadores em termos de aderência e previsão um passo a frente, além da precisão das respectivas medidas de valor em risco. Finalmente, a seção 7 tece alguns comentários finais. 


\section{Descrição dos Dados}

Nesta seção apresentamos uma descrição estatística dos dados utilizados e introduzimos a notação empregada ao longo do artigo. Utilizamos as séries de retornos diários de cotações de abertura, fechamento, máximos e mínimos, além de uma série dos retornos intradiários do IBOVESPA à vista. Classificamos as amostras da seguinte maneira: dados de retornos diários abrangendo o período de 01/08/1994 até 24/10/2001; cotações de abertura, fechamento, máximo e mínimo para o período de 01/08/1994 até 24/10/2001 e, dados de retornos intradiários cobrindo o período de 06/04/1998 a 24/10/2001. ${ }^{1}$ Os retornos intradiários foram obtidos a partir da última cotação observada em cada intervalo de 15 minutos.

\subsection{Série de retornos diários}

Particionamos a amostra dos retornos diários em dois conjuntos. O primeiro cobre o período de 01/08/1994 a 03/04/1998, e serve para a estimação dos parâmetros dos modelos da família $\mathrm{GARCH}$, enquanto que realizamos previsão um passo à frente na segunda sub-amostra (06/04/1998 a 24/10/2001). Consideramos, então, três subconjuntos da amostra para efeito de análise, as duas partições mencionadas e a amostra completa. A figura 1 apresenta, além da evolução do IBOVESPA (em log) entre agosto de 1994 e outubro de 2001, as respectivas séries de retorno e de volatilidade realizada.

A figura 1 evidencia diferentes regimes de volatilidade na primeira sub-amostra, validando a aplicação de modelos de heterocedasticidade condicional. O primeiro regime consiste em um pequeno período de baixa volatilidade, seguido por um período de alta volatilidade devido provavelmente à crise do México em dezembro de 1994. A mudança da banda de flutuação da taxa de câmbio brasileira em março de 1995 aparentemente não altera o regime de elevada volatilidade na BOVESPA. Segue-se uma nova redução da volatilidade, que volta a se elevar somente após a crise da Ásia em outubro de 1997. Percebemos ainda um período de alta volatilidade associado à moratória da Rússia em agosto de 1998, seguido de baixa volatilidade. Destacamos, finalmente, o forte impacto no grau de incerteza causado pelo ataque terrorista em 11 de setembro.

\footnotetext{
${ }^{1}$ Betina D. Martins (DEE, PUC-Rio) cedeu gentilmente uma série do IBOVESPA com amostras em intervalos de 15 minutos (fonte: CMA).
} 
Figura 1

Nível, retorno e dispersão do índice da BOVESPA
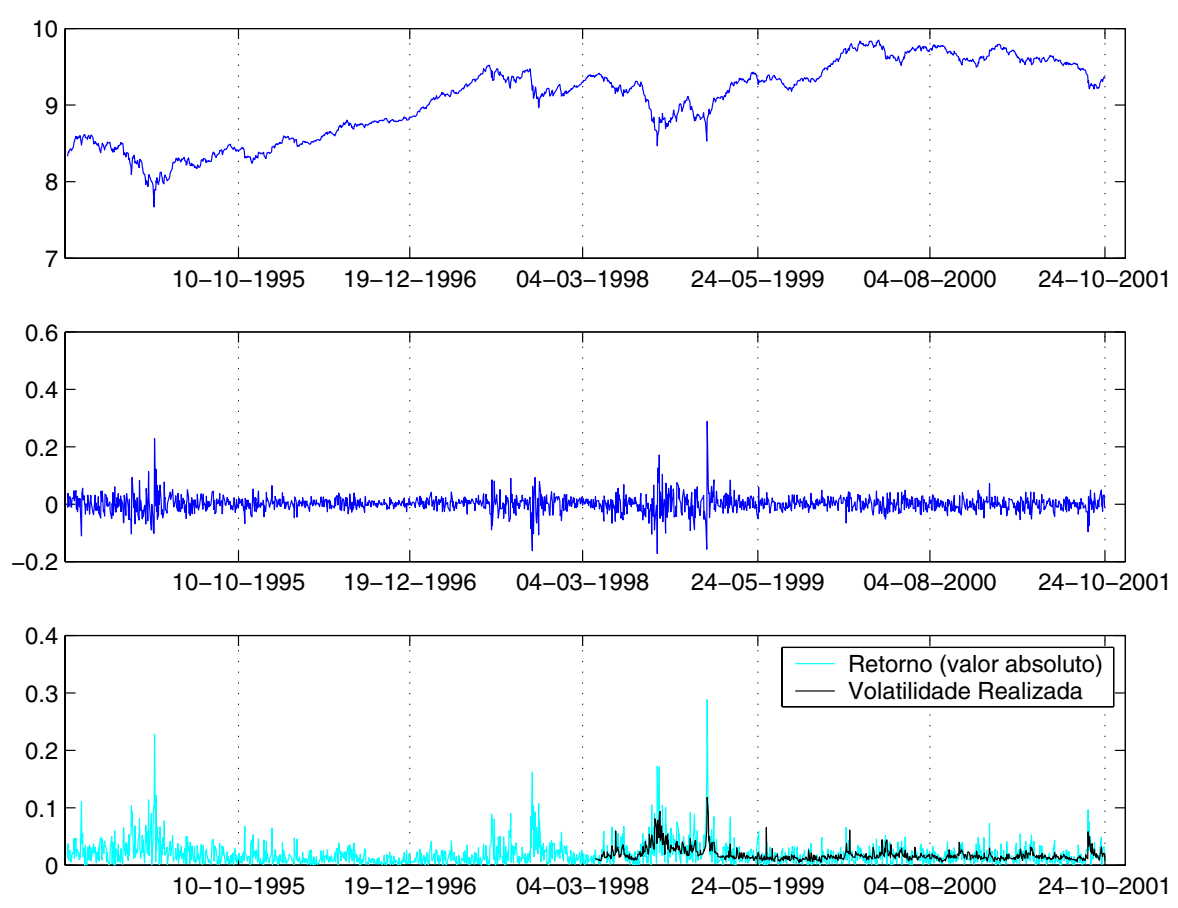

A tabela 1 documenta as principais estatísticas descritivas para as três subamostras. Em comum, todas as sub-amostras rejeitam da hipótese de normalidade. As estatísticas de Jarque-Bera e seus respectivos p-valores indicam a rejeição da hipótese nula ao nível de confiança de $99 \%$. Como a rejeição é praticamente ditada pelo excesso de curtose, a estimação de modelos de heterocedasticidade condicional se mostra natural. Encontramos ainda evidência a favor de autocorrelação tanto no primeiro subconjunto da amostra quanto na amostra inteira. A autocorrelação foi detectada tanto pela estatística $Q$ de Ljung-Box quanto pela função de autocorrelação amostral. Não encontramos nenhuma evidência de autocorrelação no segundo subconjunto da amostra. Independentemente da amostra, a função de autocorrelação amostral e a estatística $Q$ indicam evidência de autocorrelação positiva na magnitude dos retornos. 
Tabela 1

Estatísticas descritivas do retorno diário do IBOVESPA

\begin{tabular}{|c|c|c|c|c|c|c|}
\hline & \multicolumn{2}{|c|}{$08 / 94-03 / 98$} & \multicolumn{2}{|c|}{$04 / 98-10 / 01$} & \multicolumn{2}{|c|}{$08 / 94-10 / 01$} \\
\hline Média & & 0,0011 & & 0,0000 & & 0,0006 \\
\hline Mediana & & 0,0027 & & $-0,0005$ & & 0,0013 \\
\hline Desvio padrão & & 0,0276 & & 0,0289 & & 0,0282 \\
\hline Assimetria & & 0,1299 & & 0,9308 & & 0,5451 \\
\hline Curtose & & 11,2830 & & 18,5123 & & 15,1223 \\
\hline Máximo & & 0,2281 & & 0,2882 & & 0,2882 \\
\hline Mínimo & & $-0,1622$ & & $-0,1723$ & & $-0,1723$ \\
\hline Jarque-Bera & $2.603,95$ & $(0,0000)$ & $8.818,00$ & $(0,0000)$ & $10.968,43$ & $(0,0000)$ \\
\hline$Q_{1}(1)$ & 6,5167 & $(0,0107)$ & 0,0528 & $(0,8183)$ & 3,6652 & $(0,0556)$ \\
\hline$Q_{1}(6)$ & 34,9963 & $(0,0000)$ & 7,0667 & $(0,3147)$ & 27,9115 & $(0,0001)$ \\
\hline$Q_{2}(1)$ & 39,1090 & $(0,0000)$ & 77,9103 & $(0,0000)$ & 127,7993 & $(0,0000)$ \\
\hline$Q_{2}(6)$ & 302,2346 & $(0,0000)$ & 124,5584 & $(0,0000)$ & 322,9401 & $(0,0000)$ \\
\hline$Q_{2}(36)$ & 473,0041 & $(0,0000)$ & 175,2550 & $(0,0000)$ & 477,2864 & $(0,0000)$ \\
\hline
\end{tabular}

Números entre parênteses indicam p-valores. $Q_{1}(k)$ e $Q_{2}(k)$ referem-se às estatísticas de Ljung-Box para as séries de retorno e retorno ao quadrado com $k$ defasagens, respectivamente.

\section{Volatilidade Realizada}

Nesta seção, apresentamos a metodologia de extração de volatilidade desenvolvida por Andersen e Bollerslev (1998) assim como as estimativas resultantes. Recorreremos a seguinte notação. Denotaremos o logaritmo do IBOVESPA avaliado no instante $t$ por $p_{t}$. Considerando $m$ observações por dia, os retornos intradiários serão escritos como $r_{m, t} \equiv p_{t}-p_{t-\frac{1}{m}}$, onde $t=1 / m, 2 / m, \ldots$ O retorno diário é então $r_{1, t} \equiv p_{t}-p_{t-1}$. Em tempo contínuo, o retorno instantâneo é simplesmente $r_{t} \equiv \lim _{m \rightarrow \infty} r_{m, t} \equiv \mathrm{d} p_{t}$, enquanto que a sua variância instantânea é $\sigma_{t}^{2}$.

\subsection{Metodologia}

Para que possamos fazer uso dos resultados de Andersen e Bollerslev (1998), temos que fazer certas hipóteses sobre o processo gerador dos dados. Assumiremos que o IBOVESPA é gerado por uma difusão

$$
d p_{t}=\mu_{t} d t+\sigma_{t} d W_{t}
$$

em que:

$\mu_{t}$ é um componente de arrastamento càdlàg (contínuo à direita, com limite à esquerda);

$\sigma_{t}$ um processo estocástico estritamente estacionário de volatilidade, e 
$W_{t}$ um movimento Browniano padrão. O processo descrito em (1) é extremamente flexível e geral, permitindo volatilidade estocástica e um arrastamento caracterizado por saltos (Andersen et alii, 2003). Nelson (1990) mostra inclusive que, no limite em tempo contínuo, os modelos GARCH convergem para processos de difusão que são casos particulares de (1).

Por simplicidade, vamos assumir que o termo de arrastamento $\mu_{t}$ é nulo em (1). Podemos, então, escrever o preço em $t$ da seguinte maneira

$$
p_{t}=p_{0}+\int_{0}^{t} \sigma_{s} d W_{s}
$$

Os retornos amostrais intradiários, então, são

$$
r_{m, t}=p_{m, t}-p_{m, t-\frac{1}{m}}=\int_{t-\frac{1}{m}}^{t} \sigma_{s} d W_{s}=\int_{0}^{\frac{1}{m}} \sigma_{t-\frac{1}{m}+\tau} d W_{t-\frac{1}{m}+\tau}
$$

onde $t=1 / m, 2 / m, \ldots$

Assumindo independência entre $W_{t}$ e $\sigma_{t}$ e condicionando a esperança matemática no caminho amostral da volatilidade $\left\{\sigma_{s}^{2}\right\}_{s=t}^{t+1}$, temos que

$$
\mathbb{E}\left[r_{1, t+1} \mid\left\{\sigma_{s}^{2}\right\}_{s=t}^{t+1}\right]=0
$$

Ademais,

$$
\operatorname{Var}\left[r_{1, t+1}^{2} \mid\left\{\sigma_{s}^{2}\right\}_{s=t}^{t+1}\right]=\int_{t}^{t+1} \sigma_{s}^{2} d s
$$

devido à isometria de Itô. Portanto, podemos afirmar que

$$
r_{1, t+1} \mid\left\{\sigma_{s}^{2}\right\}_{s=t}^{t+1} \sim N\left(0, \Omega_{t+1}\right)
$$

onde $\Omega_{t+1} \equiv \int_{0}^{1} \sigma_{t+\tau}^{2} d \tau$ é a variância integrada. Como não é observável, precisamos estimar a variância integrada.

Assumindo que cada dia possui $m$ observações intradiárias, obtemos

$$
r_{1, t+1}^{2} \equiv\left(\sum_{j=1}^{m} r_{m, t+\frac{j}{m}}\right)^{2}=\sum_{j=1}^{m} r_{m, t+\frac{j}{m}}^{2}+2 \sum_{j=1}^{m} \sum_{i=j+1}^{m} r_{m, t+\frac{j}{m}} r_{m, t+\frac{i}{m}}
$$


Combinando (5) e (6), chega-se a

$$
\mathbb{E}\left[\left(\sum_{j=1}^{m} r_{m, t+\frac{j}{m}}\right)^{2} \mid\left\{\sigma_{s}^{2}\right\}_{s=t}^{t+1}\right]=\mathbb{E}\left(r_{1, t+1}^{2} \mid\left\{\sigma_{s}^{2}\right\}_{s=t}^{t+1}\right)=\Omega_{t+1}
$$

e, portanto,

$$
\mathbb{E}\left[\left(\sum_{j=1}^{m} r_{m, t+\frac{j}{m}}\right)^{2}\right]=\mathbb{E}\left(r_{1, t+1}^{2}\right)=\mathbb{E}\left(\Omega_{t+1}\right)
$$

Logo, $\left(\sum_{j=1}^{m} r_{m, t+\frac{j}{m}}\right)^{2}$ e $r_{1, t+1}^{2}$ são ambos estimadores não tendenciosos da variância $\Omega_{t+1}$. O estimador clássico $r_{1, t+1}^{2}$ apresenta, no entanto, um sério inconveniente. Por apresentar variância constante independentemente do tamanho da amostra, o estimador clássico é inconsistente.

Se a série de retornos não apresenta autocorrelação,

$$
\mathbb{E}\left(r_{1, t+1}^{2}\right)=\mathbb{E}\left(\sum_{j=1}^{m} r_{m, t+\frac{j}{m}}^{2}\right)
$$

onde $\hat{\sigma}_{m, t}^{2} \equiv \sum_{j=1}^{m} r_{m, t+\frac{j}{m}}^{2}$ é o estimador de variância realizada. Andersen e Bollerslev (1998) demonstram que, na ausência de autocorrelação serial, a variância realizada satisfaz

$$
\operatorname{plim}_{m \rightarrow \infty}\left(\int_{0}^{1} \sigma_{t+\tau}^{2} d \tau-\hat{\sigma}_{m, t}^{2}\right)=0
$$

Em outras palavras, a variância realizada é um estimador consistente para a variância integrada (ver Andersen et alii (2003)). Denomina-se volatilidade realizada a raiz quadrada positiva da variância realizada.

Oomen (2001) ilustra a diferença entre os dois estimadores no caso discreto, concluindo que a variância realizada $\hat{\sigma}_{m, t}^{2}$ é mais precisa que o estimador clássico $r_{1, t}^{2}$. Enquanto que o último envolve ruído na estimação da volatilidade diária, o primeiro é livre de erros de medida quando $m$ é suficientemente grande. Entretanto, se os intervalos de observação dentro do dia forem muito pequenos $(m$ muito grande), podemos ter viés na estimativa devido aos fenômenos de microestrutura (por exemplo, ausência de liquidez). Portanto, existe um problema de escolha do intervalo de amostragem. Os trabalhos empíricos na literatura situam 
a freqüência ótima entre 5 e 25 minutos. Adotamos uma freqüência de 15 minutos devido à indisponibilidade de dados intradiários em maior freqüência e ao fato dos testes de Ljung-Box indicarem autocorrelação significativa em apenas 15\% dos dias estudados.

Na verdade, a presença de autocorrelação não invalida a metodologia econométrica que utilizamos, pois Bandorff-Nielsen e Shephard (2003) demonstram a consistência da variância realizada sob condições mais gerais. Mais especificamente, eles supõem que o retorno segue um semi-martingal do tipo $r_{\infty, t}=\mu_{t}+\xi_{t}$, onde o componente de arrastamento $\mu_{t}$ tem variação limitada em qualquer subintervalo finito do intervalo $[0, \infty)$, e $\xi_{t}$ é um martingal local (por exemplo, $d \xi_{t}=$ $\left.\sigma_{t} d W_{t}\right)$. Desta maneira, permite-se que os retornos intradiários sejam correlacionados e ainda assim mantém-se a propriedade de consistência da variância realizada.

Neste artigo, escreveremos a variância realizada da seguinte maneira:

$$
\hat{\sigma}_{32, t}^{2}=\sum_{j=1}^{32} r_{32, t+j / 32}^{2}, \quad t=1, \ldots, 867
$$

onde 32 indica o número de observações intradiárias e 867 o número de dias observados. Apresentamos a volatilidade realizada na figura 1, que ilustra bem o fato que a variância do estimador clássico $r_{1, t}^{2}$ é superior a variância do estimador de variância realizada.

\section{Modelos da Família GARCH}

O objetivo desta seção é apresentar as estimativas e previsões obtidas a partir dos modelos da família GARCH. Analisamos duas formas para a equação da média condicional do retorno. Na primeira, a volatilidade não afeta a média, enquanto que, na segunda, permitimos esta possibilidade. A introdução de um termo relacionando a variância condicional com a média condicional tem um forte apelo econômico. Agentes avessos ao risco exigem uma compensação em termos de retorno, de acordo com o grau de risco do ativo.

Como encontramos sinais de dependência temporal nos retornos diários na segunda seção, especificamos um modelo auto-regressivo para a média condicional. Temos então a seguinte forma funcional

$$
r_{1, t}=\varphi+\delta r_{1, t-1}+\theta \sigma_{t \mid t-1}+\varepsilon_{t}
$$


onde $\varepsilon_{t} \mid \Psi_{t-1} \sim N\left(0, \sigma_{t \mid t-1}^{2}\right)$ e $\Psi_{t-1}$ é o conjunto de informação disponível no instante $t-1$ de tempo. ${ }^{2}$

$\mathrm{O}$ primeiro modelo define uma estrutura $\mathrm{ARCH}$ para a função cedástica. $\mathrm{O}$ segundo considera efeitos GARCH objetivando modelar de forma mais parcimoniosa a estrutura de autocorrelação. Estes dois modelos, entretanto, não capturam o efeito alavancagem segundo o qual choques negativos têm um impacto maior na volatilidade que choques positivos. Estimamos, então, modelos EGARCH e TARCH no intuito de capturar esse tipo de assimetria. Consideramos duas versões de cada modelo: $\theta$ livre e $\theta=0$ em (10). Os modelos irrestritos ( $\theta$ variando livremente) são individuados por um $M$ adicional.

Utilizamos os critérios de informação de Akaike e de Schwarz, e o teste de razão de verossimilhança para selecionar a estrutura de defasagens mais adequada. Realizamos as comparações separadamente para cada um dos modelos: ARCH(-M), GARCH(-M), EGARCH(-M) e TARCH(-M). O processo de seleção resultou nas seguintes especificações: $\operatorname{ARCH}(-\mathrm{M})(7), \operatorname{GARCH}(-\mathrm{M})(3,3), \operatorname{EGARCH}(-\mathrm{M})(3,2)$ e TARCH(-M)(1,1). Para maiores detalhes, ver Mota e Fernandes (2002).

A partir dos modelos estimados, podemos estimar a volatilidade em cada instante do tempo e realizar previsões um passo à frente. Para a análise fora da amostra, estimamos o modelo até o período $t$ e fazemos a previsão para o período $t+1$. Em $t+1$, reestimamos o modelo e fazemos a previsão para $t+2$, e assim por diante. A figura 2 apresenta a volatilidade estimada pelos modelos do tipo EGARCH(-M) utilizando a amostra completa, enquanto que a figura 3 exibe as previsões um passo à frente. ${ }^{3}$

${ }^{2}$ Como a estatística de Ljung-Box apresenta resultados diferentes de acordo ao período analisado, também estimamos recursivamente o coeficiente auto-regressivo da média condicional. Apesar das estimativas estabilizarem apenas após a desvalorização cambial em fevereiro de 1999, a variação não se mostra significativa nos níveis usuais de significância. Resolvemos, então, manter a especificação dada por (10).

${ }^{3}$ Reportamos, a seguir, apenas os resultados obtidos pelos modelos EGARCH por apresentarem o melhor desempenho na família GARCH. Os demais resultados podem ser encontrados em Mota e Fernandes (2002). 
Figura 2

Estimativas da variância
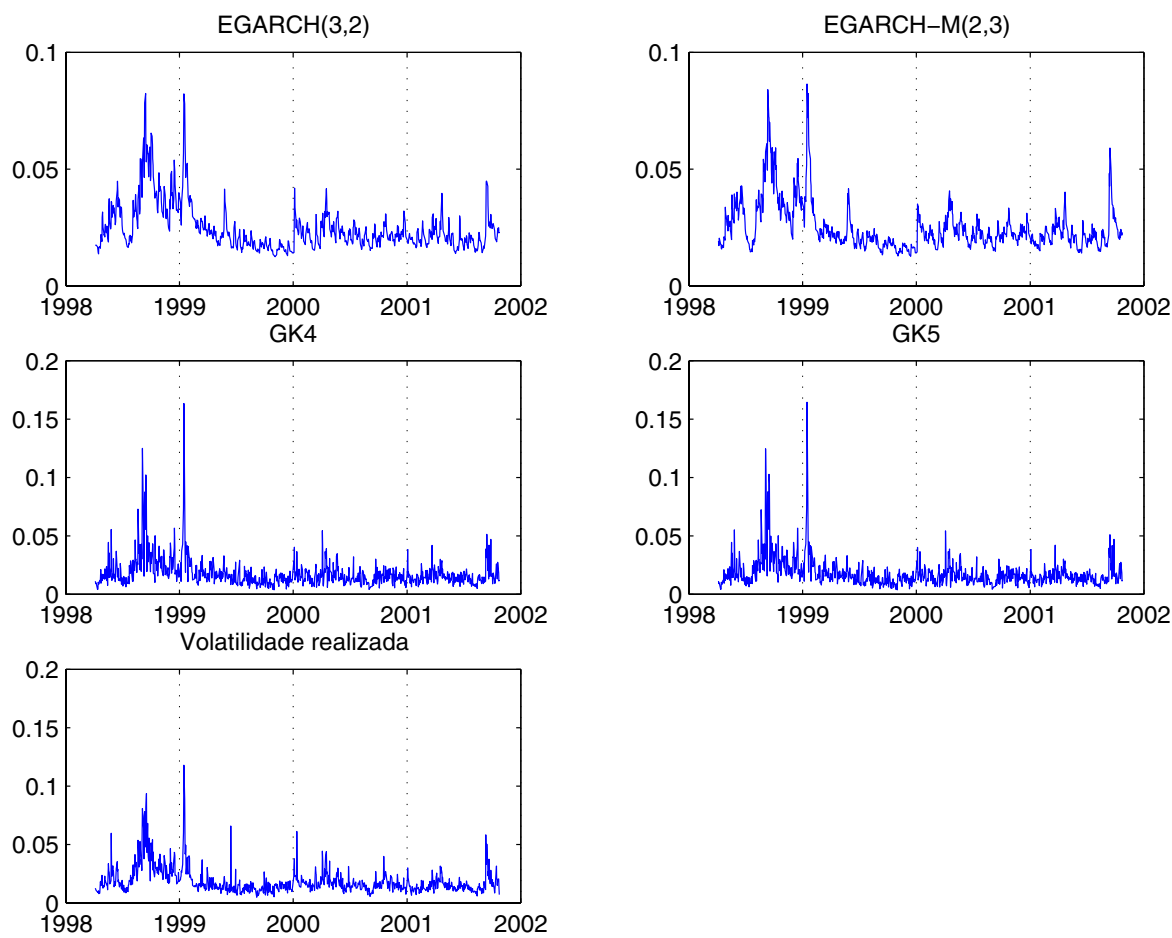

Para avaliar as diversas especificações da função cedástica, investigamos os resíduos padronizados $\hat{\eta}_{t} \equiv \hat{\varepsilon}_{t} / \hat{\sigma}_{t \mid t-1}$. Não encontramos evidência de correlação serial, indicando que o modelo auto-regressivo de primeira ordem para a média condicional é suficiente para capturar a autocorrelação do IBOVESPA. Não há evidência de correlação serial nas séries dos resíduos padronizados ao quadrado nos modelos ARCH e EGARCH, indicando que estes modelos capturam bem o agrupamento de volatilidade. Encontramos, porém, uma fraca autocorrelação de segunda ordem nos quadrados dos resíduos padronizados dos modelos GARCH e TARCH. 
Figura 3

Previsões da variância
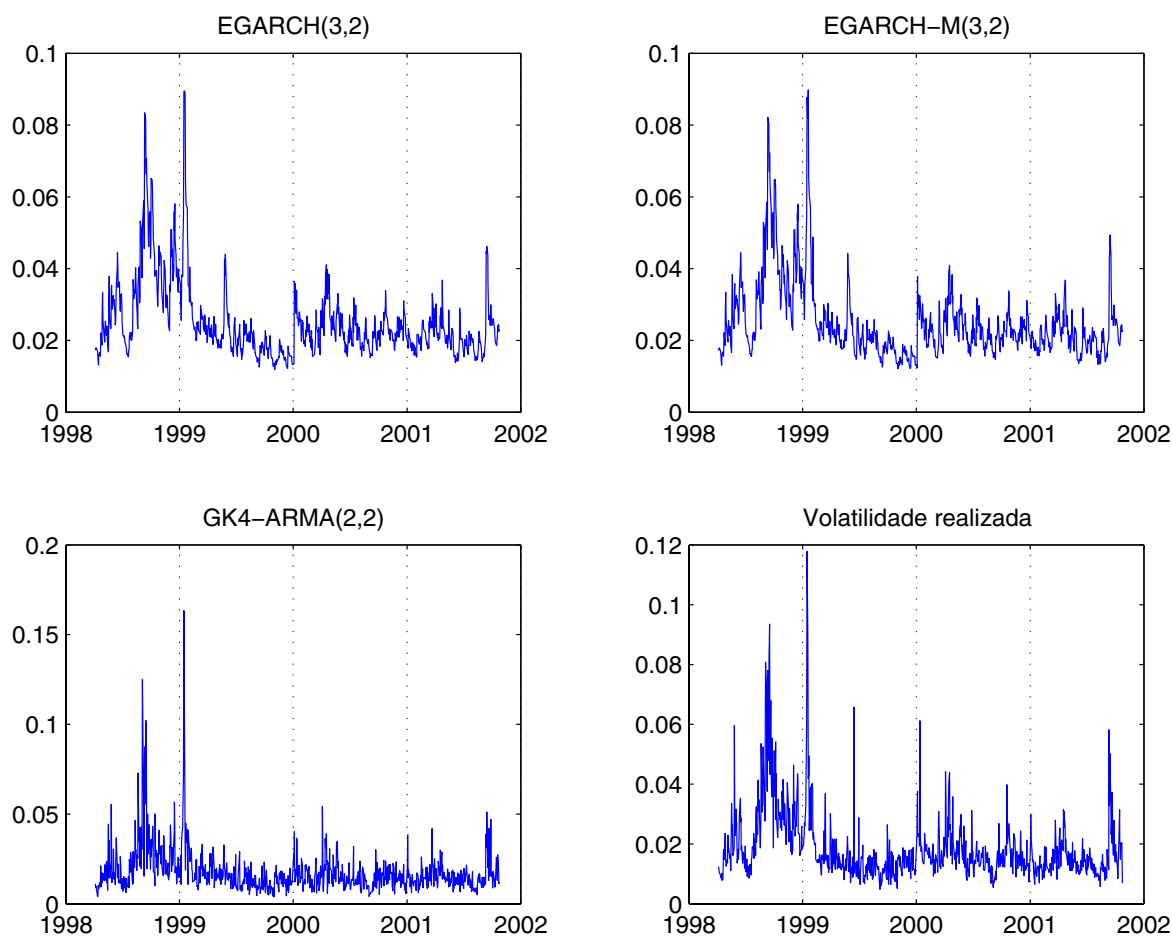

Realizamos ainda um teste de normalidade condicional dos resíduos padronizados pensando na aplicação do modelo Gaussiano de valor em risco. Em particular, testamos se $\hat{\eta}_{t} \sim N(0,1)$ através do teste de Jarque-Bera. Os resultados rejeitam a hipótese nula ao nível de significância de $5 \%$ corroborando os coeficientes de assimetria e curtose dos resíduos padronizados na tabela 2. Deste modo, calcularemos o valor em risco na seção 6.1 a partir da distribuição empírica dos resíduos padronizados em vez de assumir, como usualmente feito na literatura, normalidade.

\section{Medidas Alternativas de Volatilidade}

Apresentamos, nesta seção, os estimadores GK, que extraem medidas de volatilidade considerando apenas dados usualmente publicados na seção de finanças de jornais especializados. Garman e Klass (1980) assumem o seguinte processo gerador de dados

$$
d p_{t}=\sigma d W_{t}
$$


onde a volatilidade $\sigma$ é constante. Como (11) é uma caso particular de (1), a metodologia que empregamos para avaliar as estimativas de volatilidade é bastante natural e conveniente.

\subsection{Estimadores}

Denote por $f$ a fração do dia em que a bolsa está fechada. Sejam os preços de abertura, máximo, mínimo e fechamento do dia $t$ dados por $C_{t}, O_{t}, H_{t}$ e $L_{t}$, respectivamente. Defina ainda as cotações de máximo, mínimo e fechamento normalizadas pelo preço de abertura como $u_{t}=H_{t}-O_{t}, d_{t}=L_{t}-O_{t}$ e $c_{t}=C_{t}-O_{t}$, respectivamente.

O primeiro estimador abordado por Garman e Klass (1980) é o estimador clássico

$$
G K_{0, t} \equiv\left(C_{t}-C_{t-1}\right)^{2}=r_{1, t}^{2}
$$

Este estimador foi utilizado durante muito tempo na literatura para avaliar as estimativas derivadas de modelos do tipo GARCH por proporcionar um estimador não viesado da variância. Andersen e Bollerslev (1998) demonstram entretanto que (12) envolve considerável erro de medida.

O segundo estimador adiciona informação sobre a cotação de abertura:

$$
G K_{1, t}=\frac{\left(O_{t}-C_{t-1}\right)^{2}}{2 f}+\frac{c_{t}^{2}}{2(1-f)}
$$

Definindo a eficiência relativa de um estimador em relação ao estimador clássico $G K_{0, t}$ a partir da razão entre suas variâncias, Garman e Klass (1980) mostram que $G K_{1, t}$ traz ganhos de eficiência ao reduzir em $50 \%$ a variância da estimativa.

O terceiro estimador, atribuído a Parkinson (1980), procura aproveitar da informação contida nas cotações de mínimo e máximo assumindo que o mercado opera de forma contínua $(f=0)$ :

$$
G K_{2, t}=\frac{\left(u_{t}-d_{t}\right)^{2}}{4 \log 2}
$$

Garman e Klass (1980) demonstram que este estimador é bem mais eficiente que o anterior, pois utiliza informação sobre o que ocorreu dentro do dia e não apenas uma foto do início (ou fim) de um dia de transação. Seguindo a mesma linha de Parkinson (1980), Garman e Klass chegam a um estimador ainda mais eficiente usando não apenas as cotações de máximo e mínimo, mas também de abertura e fechamento: 


$$
G K_{3, t}=a \frac{\left(O_{t}-C_{t-1}\right)^{2}}{f}+(1-a) \frac{\left(u_{t}-d_{t}\right)^{2}}{4 \log 2(1-f)}
$$

onde $a$ é uma constante. Garman e Klass argumentam que $a=0,17$ minimiza a variância de (15).

Garman e Klass buscam ainda um estimador não viesado com menor variância na classe de estimadores invariantes à escala para $f=0$. O melhor estimador analítico invariante à escala é dado por

$$
G K_{4, t}=0,511\left(u_{t}-d_{t}\right)^{2}-0,019\left[c_{t}\left(u_{t}+d_{t}\right)-2 u_{t} d_{t}\right]-0,383 c_{t}^{2}
$$

Garman e Klass apresentam uma modificação de $G K_{4}$, que elimina os produtos cruzados em (16):

$$
G K_{5, t}=0.5\left(u_{t}-d_{t}\right)^{2}-(2 \log 2-1) c_{t}^{2}
$$

Este estimador apresenta quase a mesma eficiência que $G K_{4, t}$, mas tem a vantagem de ser mais prático.

O último estimador promove uma simples alteração em $G K_{4, t}$ para levar em consideração a fração $0<f<1$ em que o mercado se encontra fechado:

$$
G K_{6, t}=a \frac{\left(O_{t}-C_{t-1}\right)^{2}}{f}+(1-a) \frac{G K_{4, t}}{1-f}
$$

Segundo Garman e Klass (1980), $a=0,12$ minimiza a variância de (18), tornandoo mais eficiente que $G K_{4}$.

A segunda linha da figura 2 apresenta as estimativas de volatilidade obtidas a partir dos estimadores alternativos de melhor desempenho, a saber, (16) e (17). As demais estimativas evidenciam uma evolução similar, porém mais errática, da volatilidade diária. Para maiores detalhes, ver Mota e Fernandes (2002).

\subsection{Previsão um passo à frente}

O maior inconveniente dos estimadores GK é a ausência de dinâmica, em contraste com os modelos da família GARCH. Assim, para realizar previsões com base nestes estimadores, temos que assumir algum tipo de dinâmica para o processo da volatilidade. Podemos pensar nesta dinâmica como uma tentativa de captar o fluxo de informações do mercado.

As evidências de agrupamento de volatilidade sugerem o emprego de modelos do tipo ARMA: 


$$
G K_{i, t}=\omega+\sum_{j=1}^{p} \beta_{j} G K_{i, t-j}+\epsilon_{t}+\sum_{\ell=1}^{q} \alpha_{\ell} \epsilon_{t-\ell}, \quad i=0, \ldots, 6
$$

onde $\epsilon_{t}$ é um ruído branco. Experimentamos quatro modelos alternativos $[\mathrm{AR}(1)$, $\operatorname{AR}(2), \operatorname{ARMA}(1,1)$ e $\operatorname{ARMA}(2,2)]$ para a dinâmica da volatilidade conforme medida pelos estimadores GK. ${ }^{4}$ A figura 3 exibe a previsão um passo à frente com melhor performance dentre os estimadores alternativos GK: (16) com dinâmica $\operatorname{ARMA}(2,2) .^{5}$

Assim como no caso dos estimadores da família GARCH, testamos a normalidade dos resíduos padronizados. Os resultados do teste de Jarque-Bera na tabela 2 sugerem a rejeição da hipótese de normalidade dos resíduos padronizados ao nível de 5\% de significância. Em particular, os resíduos padronizados exibem leve assimetria e um elevado excesso de curtose. Ademais, notamos que os retornos padronizados dos modelos GARCH apresentam um menor excesso de curtose, mas maior assimetria, que os retornos padronizados pelos estimadores alternativos de volatilidade.

Tabela 2

Estatística descritivas dos retornos padronizados

\begin{tabular}{|c|c|c|c|c|}
\hline & \multicolumn{2}{|c|}{ Jarque-Bera } & Assimetria & Curtose \\
\hline EGARCH $(2,3)$ & 82,4896 & $(0,0000)$ & $-0,2312$ & 4,4395 \\
\hline EGARCH-M $(2,3)$ & 115,011 & $(0,0000)$ & $-0,2939$ & 4,6858 \\
\hline$G K_{4}-\operatorname{ARMA}(2,2)$ & 832,134 & $(0,0000)$ & 0,2620 & 7,7735 \\
\hline Variância realizada & 7,4322 & $(0,0243)$ & 0,1238 & 2,6197 \\
\hline
\end{tabular}

\section{Avaliação dos Estimadores de Volatilidade}

Nesta seção, confrontamos as estimativas e previsões derivadas dos modelos da família GARCH e dos estimadores alternativos tomando a variância realizada como referência. Para simplificar a exposição, omitiremos os resultados dos modelos de

\footnotetext{
${ }^{4}$ Conforme assinalado por um parecerista anônimo, os modelos da família ARMA não são teoricamente convenientes neste contexto por não garantirem, a priori, a positividade da volatilidade. A alternativa natural seria aplicar os modelos ARMA ao logaritmo das estimativas GK de volatilidade. Embora sejam um pouco mais complicadas de serem computadas, as previsões um passo à frente são qualitativamente muito semelhantes àquelas obtidas a partir de (19). Ademais, as estimativas indicam uma probabilidade extremamente baixa de violação da restrição de positividade em (19).

${ }^{5}$ Ver Mota e Fernandes (2002) para as previsões um passo à frente resultantes dos demais estimadores e dinâmicas.
} 
pior desempenho. Os resultados completos estão disponíveis em Mota e Fernandes (2002).

Adotamos três medidas de desempenho: (1) raiz do erro quadrático médio $(R E Q M)$, (2) erro absoluto médio $(E A M)$, e (3) desempenho dos estimadores quando aplicados à extração do valor em risco (VaR). Uma alternativa seria considerar o apreçamento de opções para avaliar o desempenho dos estimadores de volatilidade. A idéia consiste em montar uma carteira de opções e verificar qual estimador de volatilidade proporciona um maior retorno. Andrade (1996) realiza um estudo desse gênero para uma carteira de opções de Telebrás, concluindo que os estimadores GK não apresentam bons resultados. Entretanto, White (2000) critica exatamente esse tipo de análise por ser fortemente dependente de uma única realização do processo estocástico gerador dos dados e sugere um teste de realidade calcado em técnicas de reamostragem. ${ }^{6}$

As duas primeiras medidas de desempenho, $R E Q M$ e $E A M$, dão uma noção do quão distante estão as estimativas da variância realizada:

$$
\begin{aligned}
R E Q M(x) & =\left[\frac{1}{T} \sum_{t=1}^{T}\left(x_{t}-\hat{\sigma}_{32, t}^{2}\right)^{2}\right]^{1 / 2} \\
\operatorname{EAM}(x) & =\frac{1}{T} \sum_{t=1}^{T}\left|x_{t}-\hat{\sigma}_{32, t}^{2}\right|
\end{aligned}
$$

onde $\left\{\hat{\sigma}_{32, t}^{2}\right\}_{t=1}^{T}$ é a série de variância realizada, $T$ o número de dias na amostra, e $\left\{x_{t}\right\}_{t=1}^{T}$ a série de estimativas ou previsões da variância de um determinado modelo.

As estimativas de volatilidade mais precisas na classe GARCH advém do modelo EGARCH-M(2,3) segundo ambas as medidas. Entre os estimadores alternativos, $G K_{4}$ e $G K_{5}$ desempenham melhor de acordo aos critérios $R E Q M$ e $E A M$, respectivamente. No geral, os melhores resultados advém do modelo EGARCH$\mathrm{M}(2,3)$ e do estimador $G K_{5}$ (ver tabela 3 ). Ao considerarmos a qualidade de previsão, os modelos $\operatorname{EGARCH}(2,3)$ e $G K_{4}$-ARMA $(2,2)$ apresentam a melhor performance segundo ambas as medidas em suas respectivas classes (ver tabela 4 ). Em resumo, apesar de extremamente simples, o modelo $G K_{4}$ - $\operatorname{ARMA}(2,2)$ tem um desempenho, no mínimo, comparável aos modelos da família GARCH.

\footnotetext{
${ }^{6}$ Ver Saffi (2003) para uma aplicação do teste de realidade de White (2000) para o IBOVESPA futuro.
} 
Tabela 3

Desempenho das estimativas da variância do IBOVESPA

\begin{tabular}{lcc}
\hline Estimativa & REQM & EAM \\
\hline EGARCH $(2,3)$ & 0,0674 & 0,0387 \\
EGARCH-M $(2,3)$ & 0,0641 & 0,0385 \\
$G K_{4}$ & 0,0678 & 0,0221 \\
$G K_{5}$ & 0,0684 & 0,0221 \\
\hline
\end{tabular}

Tabela 4

Desempenho das previsões da variância do IBOVESPA

\begin{tabular}{lcc}
\hline Previsão & REQM & EAM \\
\hline EGARCH(2,3) & 0,0677 & 0,0400 \\
EGARCH-M $(2,3)$ & 0,0682 & 0,0402 \\
GK $_{4}$-ARMA $(2,2)$ & 0,0710 & 0,0289 \\
\hline
\end{tabular}

\subsection{Valor em risco}

Define-se o valor em risco (VaR) como a perda esperada máxima, em um dado horizonte de tempo, a um certo nível de confiança. Temos, portanto, dois parâmetros de escolha, o horizonte de tempo e o nível de confiança. Consideramos, a seguir, um horizonte de um dia apenas e três níveis de confiança: 90\%, 95\% e $99 \%$.

Seja $p_{1, t}$ o logaritmo do preço do ativo no dia $t$ e

$$
r_{1, t} \equiv \Delta p_{1, t}=p_{1, t}-p_{1, t-1}
$$

o seu retorno correspondente. Para calcularmos o valor em risco, precisamos especificar uma estrutura probabilística para o retorno diário. A abordagem paramétrica, por exemplo, assume usualmente normalidade condicional:

$$
r_{1, t}-\delta r_{1, t-1}-\theta \sigma_{t} \mid \Psi_{t-1} \sim N\left(0, \sigma_{t}^{2}\right)
$$

Devido à rejeição da hipótese de normalidade condicional, adotaremos uma abordagem semiparamétrica, substituindo a distribuição normal padrão pela distribuição empírica $\hat{F}$ dos resíduos padronizados. ${ }^{7}$

Definimos, então, a medida $\mathrm{VaR}_{1, t}$ como

\footnotetext{
${ }^{7}$ Ver Mota e Fernandes (2002) para os resultados da análise de valor em risco sob a hipótese de normalidade condicional. 


$$
\operatorname{Pr}\left[r_{1, t}-\delta r_{1, t-1}-\theta \sigma_{t}<-\operatorname{VaR}_{1, t} \mid \Psi_{t-1}\right]=\alpha
$$

onde $1-\alpha$ é o nível de confiança do intervalo. Seja $x_{\alpha}^{*}$ tal que $\alpha=\hat{F}\left(x_{\alpha}^{*}\right)$, $\operatorname{logo}$ $\mathrm{VaR}_{1, t}=x_{\alpha}^{*} \sigma_{t}$. Calculamos as previsões do VaR diário ao nível de confiança de 90\%, $95 \%$ e $99 \%$ para todos os modelos do tipo GARCH e estimadores GK. Apresentamos na figura 4 apenas as previsões baseadas respectivamente nos modelos EGARCH(-M) e $G K_{4}$-ARMA $(2,2)$ ao nível de confiança de confiança de $95 \%$. Os demais resultados estão disponíveis em Mota e Fernandes (2002).

Figura 4

Valor em risco
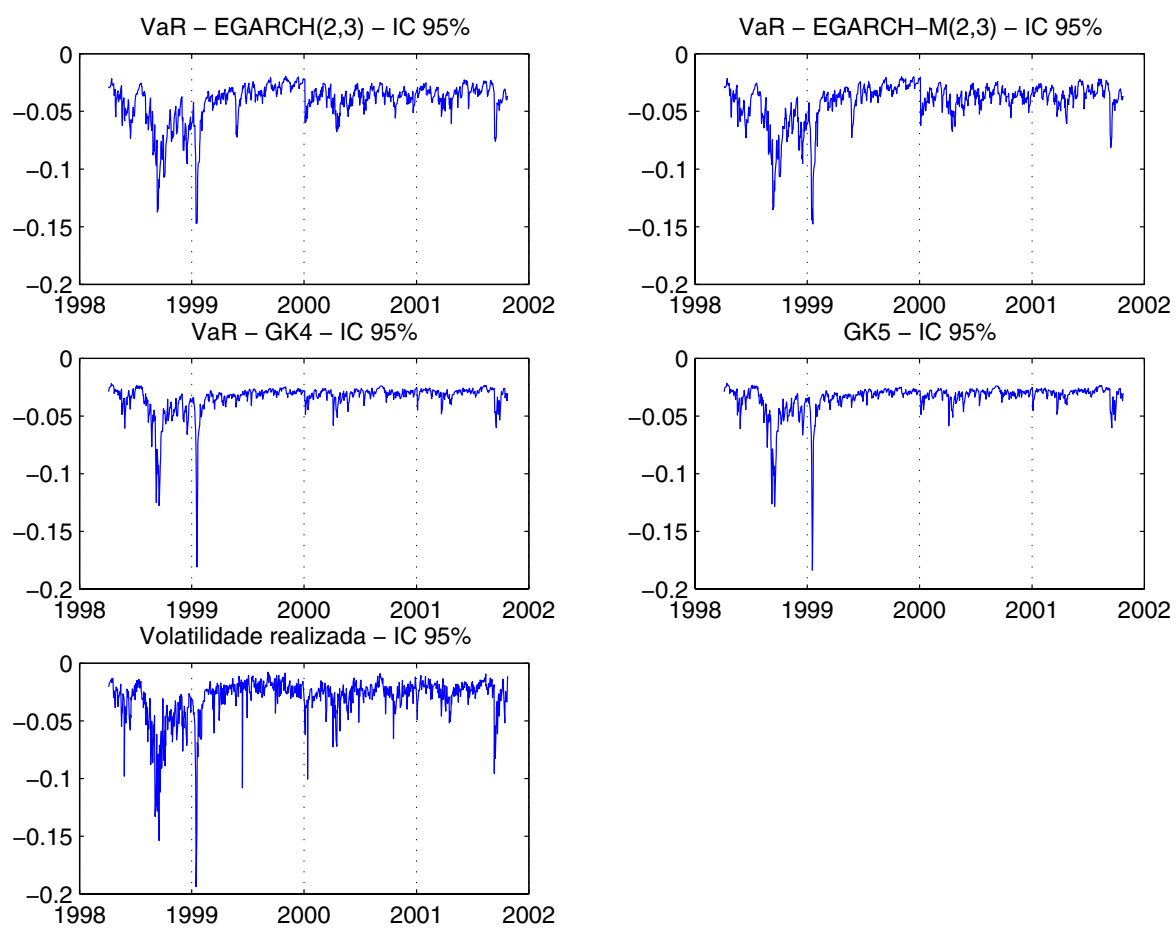

Para avaliarmos a qualidade das previsões do VaR, conduzimos um exercício de backtesting. Mais precisamente, determinamos intervalos de confiança para o número de vezes que observa-se uma perda maior do que a prevista, isto é, o número de falhas do VaR. Sob a hipótese de correta especificação do modelo, a freqüência relativa (absoluta) de falhas deve ser aproximadamente 0,10 (87 vezes), 0,05 (44 vezes) e 0,01 (9 vezes), conforme o nível de confiança.

Aos níveis de confiança de $90 \%$ e $95 \%$, os modelos EGARCH(-M) e TARCHM são os únicos com bom desempenho dentro da classe GARCH. Ao nível de 
confiança de 99\%, observamos uma melhora substancial, pois todos modelos do tipo GARCH se situam dentro do intervalo de confiança. No caso dos estimadores alternativos, $G K_{4}$ e $G K_{5}$ apresentam sempre excelentes resultados, com número de falhas dentro do esperado independentemente do nível de confiança. Ao nível de confiança de 90\%, todas as combinações estimador-dinâmica exibem um número de falhas compatível com o esperado. Ao nível de confiança de 95\%, apenas os $G K_{2}$ e $G K_{3}$ não apresentam bons resultados. Ao nível de confiança de $99 \%$, os modelos baseados em $G K_{2}, G K_{3}$ e $G K_{6}$ não se mostram adequados. Em resumo, mais uma vez, os estimadores $G K_{4}$ e $G K_{5}$ apresentam um desempenho, ao menos, comparável aos dos modelos do tipo GARCH (ver tabela 5).

Tabela 5

Desempenho das previsões da variância do IBOVESPA em termos de VaR

\begin{tabular}{lccc}
\hline \multirow{2}{*}{ Previsão } & \multicolumn{3}{c}{ Número de falhas } \\
\cline { 2 - 4 } & $90 \%$ & $95 \%$ & $99 \%$ \\
\hline EGARCH(3,2) & 89 & 43 & 9 \\
EGARCH-M(3,2) & 84 & 44 & 99 \\
TARCH(1,1) & 89 & 48 & 7 \\
TARCH-M(1,1) & 88 & 47 & 8 \\
GK 4 -ARMA & 78 & 40 & 14 \\
\hline Os intervalos de confiança de & $10 \%, 5 \%$ e $1 \%$ \\
são [72,101], [31,56] e [2,17], respectivamente.
\end{tabular}

\section{Conclusão}

Neste artigo, estimamos uma medida de volatilidade para o IBOVESPA, ainda não utilizada em trabalhos para o mercado brasileiro, denominada variância realizada. O estimador de variância realizada encerra duas propriedades importantes - ausência de viés e consistência - em contraste com o estimador clássico dado pelo retorno ao quadrado, que é inconsistente, apesar de não viesado.

Comparamos as estimativas de modelos da família GARCH com aquelas obtidas através dos estimadores GK com base na volatilidade realizada, de maneira análoga a Andersen e Bollerslev (1998). Concluímos que, em geral, os estimadores $G K_{4}$ e $G K_{5}$ apresentam resultados comparáveis aos dos modelos do tipo GARCH, apesar de serem muito mais simples. O excelente desempenho dos estimadores GK abre novos horizontes para a análise de causalidade na variância. Os modelos GARCH multivariados são extremamente difíceis de serem estimados, enquanto que uma análise calcada em variâncias realizadas exigiria uma base de dados intradiários. Os estimadores GK são facilmente calculados a partir de da- 
dos diários de fácil acesso, a saber cotações de fechamento, abertura, máximo e mínimo. Com efeito, pretendemos investigar padrões de causalidade na variância entre os principais mercados financeiros da América Latina a partir de um modelo vetor auto-regressivo para as estimativas GK.

\section{Referências}

Andersen, T. G. \& Bollerslev, T. (1998). Answering the skeptics: Yes, standard volatility models do provide accurate forecasts. International Economic Review, 39:885-905.

Andersen, T. G., Bollerslev, T., Diebold, F. X., \& Labys, P. (2003). Modeling and forecasting realized volatility. Econometrica, 71:579-625.

Andrade, S. C. (1996). Estimação da volatilidade para hedge de carteiras de opções: Um teste de eficiência. Dissertação de Mestrado, Escola de PósGraduação em Economia, Fundação Getulio Vargas.

Bandorff-Nielsen, O. E. \& Shephard, N. (2003). How accurate is the asymptotic approximation to the distribution of realised volatility? In Andrews, D., Powell, J., Ruud, P., \& Stock, J., editors, Identification and Inference for Econometric Models. A Festschrift for Tom Rothenberg. Econometric Society Monograph Series, Cambridge University Press.

Garman, M. B. \& Klass, M. J. (1980). On the estimation of security price volatilities from historical data. Journal of Business, 53:67-78.

Mota, B. S. \& Fernandes, M. (2002). Desempenho de estimadores de volatilidade na Bolsa de Valores de São Paulo. Ensaios Econômicos 458, Fundação Getulio Vargas.

Nelson, D. (1990). ARCH models as diffusion approximations. Journal of Econometrics, 45:7-38.

Oomen, R. (2001). Using high frequency stock market index data to calculate, model and forecast realized return variance. Working Paper ECO 2001/6, European University Institute.

Parkinson, M. (1980). The extreme value method for estimating the variance of the rate of return. Journal of Business, 53:61-65. 
Saffi, P. A. C. (2003). Análise técnica - sorte ou realidade? Revista Brasileira de Economia, 57:953-974.

White, H. (2000). A reality check for data snooping. Econometrica, 68:1097-1126. 